\title{
Environmental Chemistry \\ SORPTION KINETICS OF TOLUENE IN HUMIC ACID: A COMPUTATIONAL APPROACH
}

\author{
Yang-Hsin Shim, ${ }^{\dagger}$ Shian-Chee Wu, $\neq$ JyH-Shing Lin, $§$ and LiEn-Feng Lee $§$ \\ $\dagger$ Department of Soil and Environmental Sciences, Center of Nanoscience and Nanotechnology, National Chung Hsing University 250, \\ Kuo-Kuang Road, Taichung 402, Taiwan, Republic of China \\ \$Graduate Institute of Environmental Engineering, National Taiwan University 71, Chou-shan Road, Taipei 106, Taiwan, Republic of China \\ $\S$ Department of Chemistry, Tamkang University, Tamsui, Taiwan, Republic of China
}

(Received 21 December 2004; Accepted 14 July 2005)

\begin{abstract}
Molecular dynamics, a computational technique aiming to describe the time-dependent movement of molecules, has been applied to study the sorption kinetics of volatile organic contaminants in soil organic matter. The molecular dynamics simulation results obtain reasonably accurate estimates of diffusion rates and activation energy of the penetration of a volatile organic compound molecule into a model humic substance. The sorption rate of toluene to humic acid decreases with the density of the humic acid matrix and increases with temperature. All the present results indicate that the sorption of toluene into humic acid is mainly diffusion controlled. Finally, the present studies have shown that molecular dynamics of volatile organic compounds in humic substances yields meaningful results, which help in the understanding of diffusion at the molecular level and which facilitate the problemsolving capability for removing the contaminants from the soils.
\end{abstract}

Keywords-Molecular dynamics simulations Volatile organic compounds Humic acid Diffusion

\section{INTRODUCTION}

Sorption of organic molecules in humic substances is a subject of primary importance in the environmental sciences, because the sorption process contributes significantly to pollutant fate modeling and remediation of contaminated sites. Sorption kinetics of volatile organic compounds (VOCs) with different types of environmental sorbents has been studied [19]. These previous studies focused on measuring the rates of sorption in complex organomineral aggregates or humic substances at large length scales. Whereas experimental measurements of sorption rate constants can provide necessary information for soil remediation, it would be highly beneficial if we could predict the sorption rate constants by computer simulations. If this is possible, then not only can some hazardous experiments be reduced but some information about the sorption mechanisms also can be obtained [10].

Computer simulation provides a new way of studying the sorption process of VOCs in the environment. A number of researchers have applied the molecular modeling techniques to study environmental phenomena, such as the sorption mechanisms of organic contaminants adsorbed onto clays and minerals [11-15], the interactions of natural organic matter and organic contaminants [16] and inorganic contaminants [17], and the persistence of toxaphene components in mammals [18]. Increased computing power of affordable computer systems has made it possible to simulate more complicated systems using molecular dynamics. Molecular dynamics simulation, which is based on the description of molecular motion by classical mechanics, allows calculation of thermodynamic properties of the molecular system with respect to time. To our knowledge, the molecular modeling techniques rarely have been applied to study sorption kinetics and thermodynamic properties of VOCs in soil organic matter (SOM).

\footnotetext{
* To whom correspondence may be addressed (yhs@ nchu.edu.tw).
}

Therefore, in the present study, we first used the molecular dynamics simulation technique to verify our method by comparing the sorption kinetics and thermodynamic results by simulation with experimental data. Experimental diffusion coefficients and sorption enthalpies were measured using a microbalance under dry conditions in our laboratory [4]. Experimental diffusivities of three VOCs-toluene, hexane, and acetone-were quantified by the diffusion model in Aldrich humic acid (sodium form) disks. Activation energies of toluene for sorption and desorption into the humic acid disks also were determined at temperatures ranging from 25 to $45^{\circ} \mathrm{C}$ in the experiments. Next, we investigated the possible mechanism governing the sorption of toluene in humic acid matrix. Finally, we evaluated some of the thermodynamic properties of toluene in a humic acid.

\section{MATERIALS AND METHODS}

\section{Structural model and simulation method}

The chemical structure of the building block of humic acid, Temple-Northeastern-Birmingham (TNB) humic acid monomer, was described previously [19] and used to build the multiple-element structural models of humic acid [20]. The average structural unit of humic acid, the proposed TNB structure, was obtained from chemical analysis by Davis et al. [19]. Both Davis et al. and Sein et al. [20] indicated that chemical studies suggest TNB humic acid building blocks link through amide bonds. The structural properties of the humic acid model were in agreement with the real experimental conditions [20]. The basic building block structure of humic acid was produced in the present study using the Cerius ${ }^{2}$ Builder (Molecular Simulation, San Diego, CA, USA). A stable confirmation of the humic acid model, consisting of eight monomers, was built by gradually adding one monomer after another; at the same time, energy minimization calculations were executed. Finally, the target compound (i.e., the toluene molecule) was inserted into 


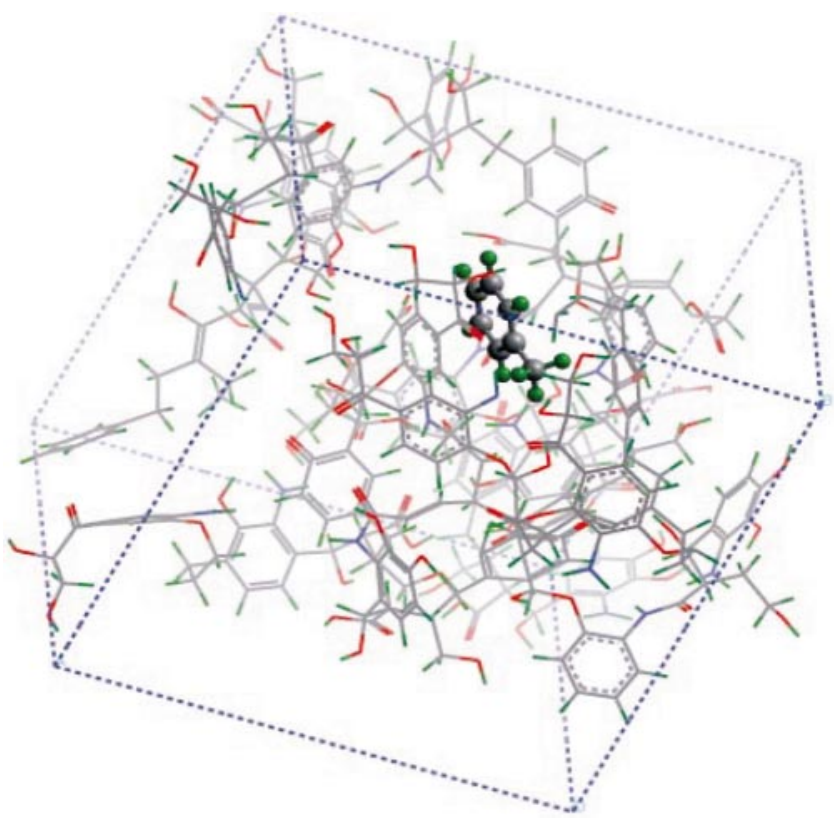

Fig. 1. The lowest-energy humic acid model containing a toluene molecule (the ball-and-stick form). Carbon atoms are gray, nitrogen atoms blue, oxygen atoms red, and hydrogen atoms green.

the open space formed among chains within the humic acid to establish our structural model, as shown in Figure 1. The unit-cell dimensions are $19.95 \times 20.37 \times 20.87 \AA$, and $\alpha=$ $85.60^{\circ}, \beta=88.53^{\circ}$, and $\gamma=93.87^{\circ}$. Different density structures were obtained by performing energy minimization gradually after each dimension of the unit cell of the humic acid structure was reduced slightly.

The molecular dynamics technique involves calculating the time evolution at an atomic level for an ensemble of particles acting under specified interatomic forces by numerically solving the equation of motion in an iterative manner. The interatomic force parameters used for toluene and humic acid throughout all the molecular dynamics simulations were taken from the universal force field described by Rappe et al. [21] and distributed by Molecular Simulation Inc. (San Diego, CA, USA). Atomic charges were calculated using the $Q_{\text {eq }}$ method [22]. A cutoff distance of $8.5 \AA$ was used for the van der Waals interactions. The Ewald summation technique was employed for the calculation of the intermolecular coulombic potential. All the calculations were performed on SGI Origin 2000/3800 workstations at the National Center for High-Performance Computing in Hsingchu, Taiwan, Republic of China.

Our simulation procedure was as follows: First, simulations of the structural model of humic acid plus toluene were run using the Dynamic module of the Cerius2 molecular modeling suite with the NVT ensemble and the periodic boundary conditions. In the NVT ensemble, the number of molecules $(\mathrm{N})$, volume (V), and temperature of the system (T) are kept constant. The Open Force Field module was used as the simulation engine. These simulations at 300, 350, and $400 \mathrm{~K}$ were equilibrated for 50 picoseconds (ps) and then run for $200 \mathrm{ps,} \mathrm{when}$ data were collected for mean-squared displacement of the migrations. The constant time step of 1 femtosecond (fs) is taken for all the simulations of the present study. The temperature was maintained by coupling the system to a temperature bath using the Nose-Hoover approach [23]. From the molecular dynamics trajectories of the system, we were able to calculate the diffusion coefficient via the Einstein relationship, which is discussed below.

\section{Dynamics}

The diffusion coefficients are related to the time-dependent, mean-squared displacement and can be calculated according to statistical mechanical principles [24,25]. In short, the diffusion coefficient was extracted from the proportionality constant according to the Einstein form of diffusion

$$
D=\frac{1}{6} \lim _{t \rightarrow \infty} \frac{d}{d t}\left\langle\left[r_{i}(0)-r_{i}(t)\right]^{2}\right\rangle
$$

where $D$ is the diffusion coefficient and $r_{i}(t)$ is the position vector of penetrant $i$ at time $t$.

Considering a binary system consisting of the polymerized humic acid and the penetrant toluene molecule, the meansquared displacement from the dynamics trajectory was equated to the average of the displacement in Equation 1 for the calculation of the diffusion coefficient. The temperature dependence of diffusion coefficient can be described by the Arrhenius equation $[4,6,26]$

$$
\frac{d \ln D}{d t}=\frac{E}{R T^{2}}
$$

where $E$ is the activation energy of the diffusion process, $R$ is the ideal gas constant, and $T$ is the absolute temperature. The apparent diffusional activation energy can be estimated by plotting $\ln D$ versus $1 / T$.

\section{RESULTS AND DISCUSSION}

\section{Sorption kinetics of VOCs in humic acid model}

The humic acid model, which is based on the combination of the average structural unit of humic acid obtained from chemical analysis and modeling by Sein et al. [20], was proposed to study sorption kinetics of VOCs, with toluene as a model compound (Fig. 1). Sein et al. indicated a link between the amine and carboxylate of these molecules, which generates a stable helical structure. Our structural model of humic acid has a feature very similar to their helical humic acid structure. The toluene was inserted at three different positions to get the lowest energy conformation before the equilibration step in the simulation procedure.

Li et al. [10] used 10 to 20 ps to obtain the diffusion coefficients of chemicals in amorphous polymers. We examined the effect of the simulation time on the calculated values of diffusion coefficient. The calculated value of the diffusion coefficient approached a steady value as the simulation time became longer, and it reached a stable lowest value at approximately 200 ps (Fig. 2).

The averaged diffusivity of toluene in humic acid estimated from the collected trajectories in the molecular dynamics simulations using Equation 1 was $2.92 \times 10^{-8} \mathrm{~cm}^{2} / \mathrm{s}$ at $296 \pm 8.7$ $\mathrm{K}$ (mean \pm standard deviation). Compared to the experimental diffusivity of $6.6 \times 10^{-9} \mathrm{~cm}^{2} / \mathrm{s}$ for sorption and $1.2 \times 10^{-9}$ $\mathrm{cm}^{2} / \mathrm{s}$ for desorption at $298 \mathrm{~K}$ estimated by Chang et al. [4], the value in the present study was approximately one order of magnitude larger than the experimental results. Because of the slight inconsistency between our calculated and experimental diffusion coefficients, we compared the density used for the modeled humic acid and that measured for the humic acid used in the experiment. The modeled humic acid had a density of $1.08 \mathrm{~g} / \mathrm{cm}^{3}$, and the experimental humic acid had a density of $1.23 \mathrm{~g} / \mathrm{cm}^{3}$ [4]. 


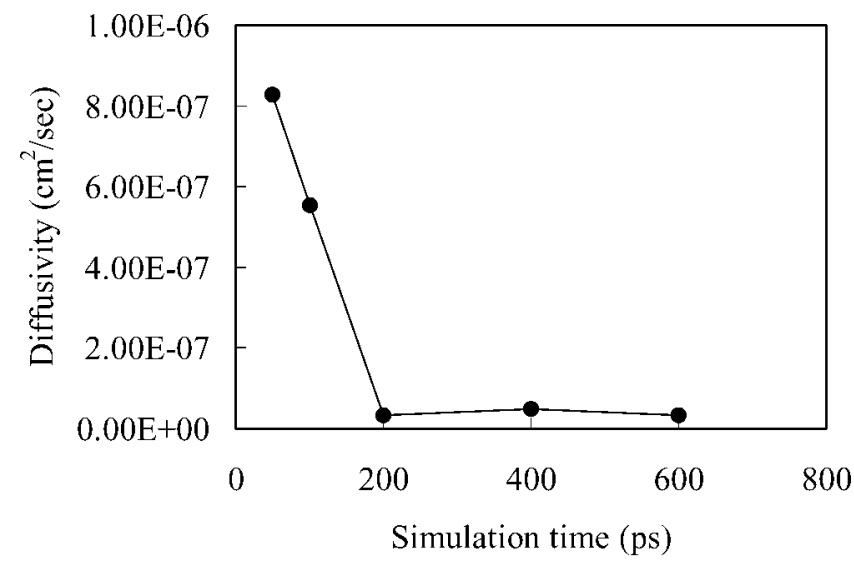

Fig. 2. Effect of simulation time on diffusivity of toluene in the humic acid model.

The calculated diffusion coefficient values are functions of the density of these humic acid models (Fig. 3). The toluene molecule has lower diffusion coefficients at higher densities of humic acid structures. The same trend is observed for several chemicals in two polymers [10]. Penetrants are expected to move faster in a looser network of the same humic acid polymer, because the void volume of the polymer matrix provides the diffusional pathway for the penetrants.

The averaged diffusivity of toluene in humic acid at a density of $1.20 \mathrm{~g} / \mathrm{cm}^{3}$ was $8.43 \times 10^{-9} \mathrm{~cm}^{2} / \mathrm{s}$ at $297 \pm 9.5 \mathrm{~K}$. A higher density of the polymer would result in a lower mobility of the penetrants and, thereby, a decreased diffusivity. Compared to the experimental diffusivity of toluene in humic acid under dry conditions at $298 \mathrm{~K}$ [4], the value in the present study was on the same order of magnitude as the experimental results.

Based on the results of molecular simulation in the present study and in comparison to experimental results reported in the literature [4-7], we propose that the VOC sorption rates measured in some previous sorption work [4-7] were controlled by diffusion of VOC molecules in the humic substance matrix. Also, the value of the diffusivity in humic substances was far less than that in air or in water and, instead, was more like that in synthetic polymers. Furthermore, the apparent diffusivities of toluene into soil humic substances (humic acid, fulvic acid, and humin) are on the order of approximately $10^{-9}$ $\mathrm{cm}^{2} / \mathrm{s}$ [4-7]. Holmen and Gschwend [27] reported the thick-

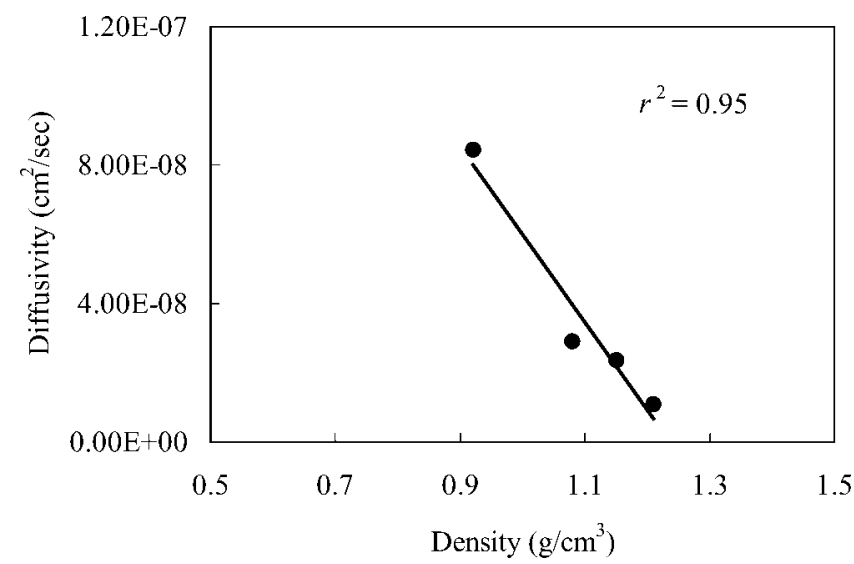

Fig. 3. Effect of the density of humic acid models on diffusion coefficients.
Table 1. Calculated diffusion coefficients of toluene in the humic acid model at three different temperatures

\begin{tabular}{lc}
\hline Temperature $(\mathrm{K})$ & Diffusivities $\left(\mathrm{cm}^{2} / \mathrm{s}\right)$ \\
\hline $297 \pm 9.5$ & $8.43 \times 10^{-9} \pm 6.12 \times 10^{-9}$ \\
$346 \pm 10$ & $6.36 \times 10^{-8} \pm 9.22 \times 10^{-8}$ \\
$394 \pm 11$ & $3.82 \times 10^{-7} \pm 5.26 \times 10^{-7}$ \\
\hline
\end{tabular}

ness of humic substances coating three sandy aquifers was approximately 10 to $100 \mu \mathrm{m}$. Piatt and Brusseau [5] suggested that the organic coating thickness was approximately $20 \mu \mathrm{m}$ for two soils. According to these length scales in soils and the diffusivity, the time that toluene molecules need to penetrate a SOM matrix could be only a few minutes in the environment.

\section{Thermodynamics}

The average diffusivities of $6.36 \times 10^{-8} \mathrm{~cm}^{2} / \mathrm{s}$ at approximately $350 \mathrm{~K}$ and $3.82 \times 10^{-7} \mathrm{~cm}^{2} / \mathrm{s}$ at approximately 400 $\mathrm{K}$ are shown in Table 1 and Figure 4 . The diffusivity of toluene increases with temperature, which is consistent with the experimental results of Chang et al. [4]. A higher temperature will increase the rate of diffusion because of an increase in energy that facilitates the displacement of polymer segments and helps the mobility of VOC molecules. These influences also can be observed in the results of the molecular dynamics simulation.

As shown in Figure 4 and estimated by Equation 2, an activation energy barrier of $7.8 \mathrm{kcal} / \mathrm{mol}$ is required when toluene molecules are squeezing through the macromolecular matrix. This value is slightly lower than the activation energies of toluene at $10.1 \mathrm{kcal} / \mathrm{mol}$ for sorption and at $15.7 \mathrm{kcal} / \mathrm{mol}$ for desorption into the humic acid at 15 to $45^{\circ} \mathrm{C}$ [4].

The reason for the observed difference between the activation energy in the simulation and the experimental study is that there would be reduced hindrance for vapor movement in a dehydrated humic acid network. The humic acid used in the previous experimental work by Chang et al. [4] was dried with nitrogen gas; nevertheless, some water molecules persisted in the humic acid matrix. However, the humic acid for the simulation did not contain any water molecules. Dehydrating the humic acid with water increases the partitioning capacity of the humic acid for nonpolar organic compounds, an indication

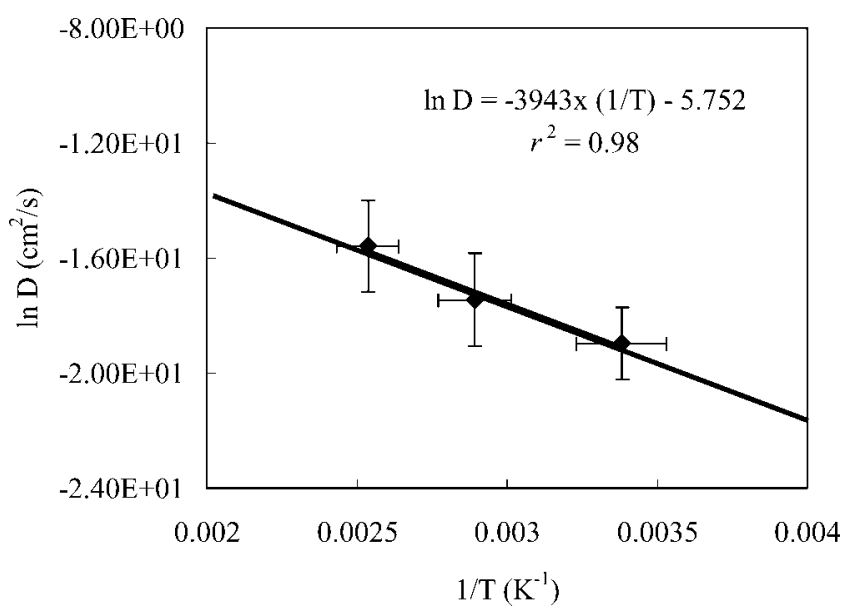

Fig. 4. The $\ln D$ versus $1 / T(\mathrm{~K})$ for toluene sorption in the humic acid model. Temperature ranges from 300 to $400 \mathrm{~K}$. Error bars represent one standard deviation of triplicates in the simulations. 
Table 2. Calculated diffusion coefficients of toluene in the waterhumic acid model at three different temperatures

\begin{tabular}{lc}
\hline Temperature $(\mathrm{K})$ & Diffusivities $\left(\mathrm{cm}^{2} / \mathrm{s}\right)$ \\
\hline $298 \pm 8$ & $4.22 \times 10^{-9} \pm 3.16 \times 10^{-9}$ \\
$345 \pm 10$ & $4.77 \times 10^{-8} \pm 2.66 \times 10^{-8}$ \\
$396 \pm 10$ & $2.00 \times 10^{-7} \pm 1.21 \times 10^{-7}$ \\
\hline
\end{tabular}

that the overall polarity of the hydrated humic acid is decreased $[28,29]$. As a result of the humic acid dehydration, the humic acid molecular network might become less cross-linked. Schaumann and Leboeuf [30] also indicated that water-based cross-links in a SOM matrix decreased with decreasing water content. As a consequence, the diffusional activation energy of toluene within the molecular humic acid model that is used should be smaller than the value estimated from humic acid under typical environmental conditions.

\section{Diffusion of VOCs in a water-humic acid model}

Different numbers of water molecules were inserted into the humic acid model. Each of these water-humic acid models was re-equilibrated by energy minimization, and dynamic simulation procedures were performed. Increasing the number of water molecules up to five in the humic acid model, the order of magnitude of the diffusivity was kept at approximately $10^{-9}$ $\mathrm{cm}^{2} / \mathrm{s}$. After adding six water molecules in the model, the average diffusivity of replicates at $300 \mathrm{~K}, 1.02 \times 10^{-14} \mathrm{~cm}^{2} / \mathrm{s}$, was far from the experimental data $\left(10^{-9} \mathrm{~cm}^{2} / \mathrm{s}\right)$. For the model with seven water molecules, the diffusivity was $2.65 \times 10^{-16}$ $\mathrm{cm}^{2} / \mathrm{s}$. The diffusivity was far less than the experimental measurements after adding more than five water molecules. The water-humic acid model including five water molecules was selected to investigate the sorption kinetics of VOC molecules.

In Table 2, the averaged diffusivity of toluene in the waterhumic acid model is $4.22 \times 10^{-9} \mathrm{~cm}^{2} / \mathrm{s}$ at $300 \mathrm{~K}$. This value is close to the averaged experimental data that were estimated in our laboratory [4]. Because of the hydrogen-bond formation between polar functional groups and water molecules, the humic acid molecular network might become more cross-linked. Schaumann and Leboeuf [30] also found that water molecules may increase the degree of the cross-links in SOM matrix because of the formation of hydrogen bond-based cross-links between water molecules and SOM side chains. The averaged diffusivities at three different temperatures are shown in Table 2 and Figure 5. The activation energy of toluene into waterhumic acid is approximately $9.3 \mathrm{kcal} / \mathrm{mol}$. The energy barrier increases after adding water molecules. The hindrance of water molecules on the mobility of toluene molecule was observed in the computer dynamic simulations. The mobility of toluene molecule is hindered by water molecules and the cross-linked structure because of the hydrogen bonding of water molecules and polar functional groups of humic acid.

The diffusivities of organic chemicals in some polymers are $1.8 \times 10^{-7} \mathrm{~cm}^{2} / \mathrm{s}$ for toluene in butyl rubber at $30^{\circ} \mathrm{C}$ [31] and $5.2 \times 10^{-7} \mathrm{~cm}^{2} / \mathrm{s}$ and $1.7 \times 10^{-7} \mathrm{~cm}^{2} / \mathrm{s}$ for benzene and $o$-xylene, respectively, in natural rubber at $25^{\circ} \mathrm{C}$ [32]. The complicated cross-linked network of humic acid may cause the smaller diffusivities of VOCs than are found in these polymers.

Li et al. [10] and Wang [33] have done molecular dynamics simulation of organic molecular diffusion in amorphous polymers. The difference between the calculated and experimental

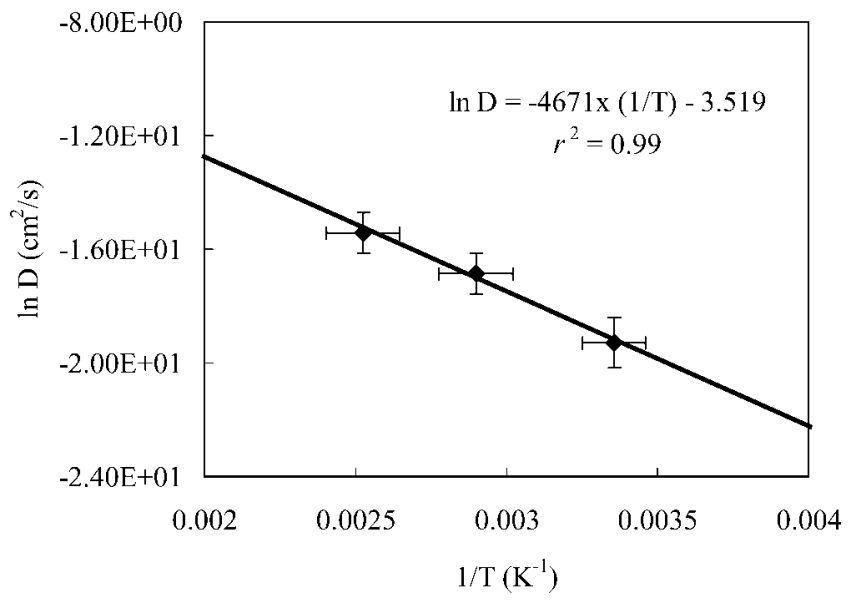

Fig. 5. The $\ln D$ versus $1 / T(\mathrm{~K})$ for toluene sorption in the waterhumic acid model. Temperature ranges from 300 to $400 \mathrm{~K}$. Error bars represent one standard deviation of triplicates in the simulations.

diffusion coefficients was as large as 24,000-fold [10]. The accuracy of calculations and the correlation with experimental values could be improved by using the different force field parameters, simulation time, and size of molecular models. Furthermore, the computer simulation should reflect exactly the experimental system and condition, so the density and water effect were considered for the humic acid model in the present study.

The similar trend between the molecular simulation and the macroscopic experimental results show that the molecular dynamics method can mimic molecular motion in actual humic acid systems.

Acknowledgement-The authors gratefully acknowledge the financial support of the National Science Council of Taiwan, Republic of China (contracts NSC 91-2211-E-002-008, NSC 92-2211-E-002-027, and NSC 93-2313-B-005-088).

\section{REFERENCES}

1. Ball WP, Robert PV. 1991. Long-term sorption of halogenated organic-chemicals by aquifer material. 2. Intraparticle diffusion. Environ Sci Technol 25:1237-1249.

2. Steinberg SM, Pignatello JJ, Sawney BL. 1987. Persistence of 1,2-dibromoethane in soils: Entrapment in intraparticle micropores. Environ Sci Technol 21:1201-1208.

3. Wu S, Gschwend PM. 1986. Sorption kinetics of hydrophobic organic compounds to natural sediments and soils. Environ Sci Technol 20:717-725.

4. Chang M, Wu S, Chen C. 1997. Diffusion of volatile organic compounds in pressed humic acid disks. Environ Sci Technol 31: 2307-2312.

5. Piatt JJ, Brusseau ML. 1998. Rate-limited sorption of hydrophobic organic compounds by soils with well-characterized organic matter. Environ Sci Technol 32:1604-1608.

6. Shih Y, Wu S. 2002. Kinetics of toluene sorption in humin under two different levels of relative humidity. J Environ Qual 31:970978.

7. Shih Y, Wu S. 2002. Sorption kinetics of selected volatile organic compounds in humin. Environ Toxicol Chem 21:2067-2074.

8. Shih Y, Wu S. 2004. Kinetics of toluene sorption and desorption in $\mathrm{Ca}$ - and $\mathrm{Cu}$-montmorillonites investigated with FTIR spectroscopy under two different levels of humidity. Environ Toxicol Chem 23:2061-2067.

9. Shih Y, Wu S. 2005. Distinctive sorption mechanisms of soil organic matter and mineral components as elucidated by organic vapor uptake kinetics. Environ Toxicol Chem 24:2827-2832.

10. Li T, Kildsig DO, Park K. 1997. Computer simulation of molecular diffusion in amorphous polymers. $J$ Control Release 48:57-66.

11. Kubicki JD, Itoh MJ, Schroeter LM, Apitz SE. 1997. Bonding 
mechanisms of salicylic acid adsorbed onto illite clay: An ATRFTIR and molecular orbital study. Environ Sci Technol 31:11511156.

12. Teppen BJ, Yu CH, Miller DM, Schäfer L. 1998. Molecular dynamics simulations of sorption of organic compounds at the clay mineral/aqueous solution interface. Journal of Computational Chemistry 19:144-153.

13. Boyd SA, Sheng G, Teppen BJ, Johnston CT. 2001. Mechanism for the adsorption of substituted nitrobenzenes by smectite clays. Environ Sci Technol 35:4227-4234.

14. Farrell J, Luo J, Blowers P, Curry J. 2002. Experimental and molecular mechanics and ab initio investigation of activated adsorption and desorption of trichloroethylene in mineral micropores. Environ Sci Technol 36:1524-1531.

15. Luo J, Farrell J. 2003. Examination of hydrophobic contaminant adsorption in mineral micropores with grand canonical Monte Carlo simulations. Environ Sci Technol 37:1775-1782.

16. Kubicki JD, Apitz SE. 1999. Model of natural organic matter and interactions with organic contaminants. Organic Geochemistry 30:911-927.

17. Nantsis EA, Carper WR. 1998. Effects of hydration on the molecular structure of magnesium-fulvic acid complexes: A MOPAC (PM3) study. J Mol Struct (Theochem) 468:51-58.

18. Vetter V, Scherer G. 1999. Persistency of toxaphene components in mammals that can be explained by molecular modeling. Environ Sci Technol 33:3458-3461.

19. Davis G, Fataftah A, Cherkassiy A, Ghabbour EA, Radwan A, Jansen SA, Kolla S, Paciolla MD, Sein LT Jr, Buermann W, Balasubramanian M, Budnick J, Xing B. 1997. Tight metal binding by humic acids and its role in biomineralization. $J$ Chem Soc Dalton Trans 21:4047-4060.

20. Sein LT Jr, Varnum JM, Jansen SA. 1999. Conformational modeling of a new building block of humic acid: Approaches to the lowest energy conformer. Environ Sci Technol 33:546-552.

21. Rappe AK, Casewit CJ, Colwell KS, Goddard WA, Skiff WM. 1992. UFF, a full periodic table force field for molecular me- chanics and molecular dynamics simulations. J Am Chem Soc 114:10024-10035.

22. Rappe AK, Goddard WA. 1991. Charge equilibrium for molecular dynamics simulations. Journal of Physical Chemistry 95:33583363.

23. Hoover WG. 1985. Canonical dynamics: Equilibrium phase space distributions. Physical Review A 31:1695-1697.

24. Haile JM. 1997. Molecular Dynamics Simulation: Elementary Methods. John Wiley, New York, NY, USA.

25. Theodorou DN. 1996. Molecular simulations of sorption and diffusion in amorphous polymers. In Neogi P, ed, Diffusion in Polymers. Marcel Dekker, New York, NY, USA, p 67.

26. Crank J, Park GS. 1968. Diffusion in Polymers. Academic, London, UK.

27. Holmen BA, Gschwend PM. 1997. Estimating sorption rates of hydrophobic organic compounds in iron oxide- and aluminosilicate clay-coated aquifer sands. Environ Sci Technol 31:105113.

28. Chiou CT, Kile DE, Malcolm RL. 1988. Sorption of vapors of some organic liquids on soil humic acid and its relation to partitioning of organic compounds in soil organic matter. Environ Sci Technol 22:298-303.

29. Chiou CT. 2002. Partition and Adsorption of Organic Contaminants in Environmental Systems. John Wiley, New York, NY, USA.

30. Schaumann GE, Leboeuf EJ. 2005. Glass transitions in peat: Their relevance and impact of water. Environ Sci Technol 39:800-806.

31. Schneider NS, Moseman JA, Sung N. 1994. Toluene diffusion in butyl rubber. Journal of Polymer Science Part B: Polymer Physics 32:491-499.

32. Guo CJ, De Kee D, Harrison B. 1995. Free volume model and diffusion of organic solvents in natural rubber. Journal of Applied Polymer Science 56:823-829.

33. Wang JY. 2002. Diffusion of drug molecules in polymeric membranes-Molecular dynamics simulations and free volume theory analysis. PhD thesis. National Taiwan University, Taipei, Taiwan, Republic of China. 